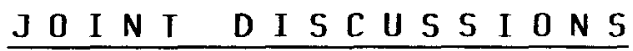




\section{REFERENCE FRAMES}

\section{Scientific Organizing Committee}

J. HUGHES (Chairman)

H. EICHHORN, W. FRICKE, K. JOHNSTON, J. KOVALEVSKY,

D. McCARthy, I. MUeller, A. MURRAY, M. MiYamoto, h. SCHOLl

Supporting Commissions : 4, 7, 8, 19, 20,

$24,31,33,40$ 
USES AND REQUIRED CHARACTERISTICS OF REFERENCE FRAMES

FOR GALACTIC ASTRONOMY

\author{
Roland Wielen \\ Astronomisches Rechen-Institut \\ Heidelberg, Germany
}

\title{
ABSTRACT
}

Galactic astronomy requires mainly accurate proper motions of stars, referred to a system which represents an inertial system as closely as possible. We discuss the required accuracy, give an outlook on forthcoming reference frames of use for galactic astronomy, and discuss observationally determined upper limits on the rotation of the extragalactic reference frame.

\section{INTRODUCTION}

An astronomical reference frame provides in most cases information about two types of quantities, namely positions and proper motions. Positional information of highest accuracy is very rarely used in galactic astronomy. In contrast, proper motions with respect to an inertial system are of extreme importance for a large variety of investigations on our Galaxy.

\section{POSITIONS}

Accurate positions of stars and other objects are mainly used for the identification of the same object at different wavelengths, e.g. in a comparison of optical and radio observations of galactic sources. Such a comparison of positions at different wavelengths is, however, more frequently required for extragalactic astronomy (e.g. for quasars) than for galactic studies.

The requirements which celestial reference frames have to meet for such identification purposes, are relatively weak: The main requirement is the absence of a relative off-set between the reference systems at the different wavelengths. It is especially unnecessary for identification purposes that the reference frame represents an inertial system. 


\section{PROPER MOTIONS}

Proper motions of stars are extensively used in galactic astronomy. There are two, rather distinct types of applications of proper motions (Wielen 1982). Either one is interested in the collective motions of stars: An example is the determination of the basic galactic rotation, i.e. of the Oort constants $A$ and $B$ and of the local frequency of galactic rotation, $\omega_{0}=A-B$. Other examples are investigations of streaming motions of stars of various ages, connected with spiral arms and predicted by the density-wave theory, or the determinations of the stream parallax of the Hyades, using the convergent-point method. In the second kind of applications of proper motions, one is interested in deriving individual space velocities of stars. An example for such investigations is the determination of the birth place of a star by following its orbit backwards in time, starting from its present position with the observed space velocity.

Nearly all applications in galactic astronomy require in principle that the proper motions do actually refer to an inertial system. For the determination of galactic rotation, for example, it is obviously desirable that the reference frame itself does not show any spurious rotation. The required accuracy for the link of the reference frame to an inertial system can best be judged from a comparison with the value of the basic galactic rotational frequency, $\omega_{0}=A-B \sim 0: 5 /$ century $=5$ marcsec/year. It is clear that for a good determination of $\omega_{0}$, the spurious rotation of the reference frame should be significantly smaller than 1 marcsec/ year.

For many determinations of streaming motions of stars, including the stream parallax of the Hyades, the rigidity of the reference frame is very important. This means that the system of proper motions should be as far as possible free from regional distortions which can mimic streaming motions of stellar ensembles.

The typical mean error of the FK4 system is supposed to be of the order of $\pm 0: 1 /$ century $=1 \mathrm{marcsec} /$ year . This translates into a typical systematic error in velocity of $\pm 5 \mathrm{~km} / \mathrm{s}(\mathrm{r} / 1 \mathrm{kpc})$, where $r$ is the distance of the object.

An extremely important property of a reference frame is its practical applicability. This means that proper motions with respect to such a reference frame should exist, or should be easily obtainable, for a significant number of interesting objects. Presently, we are in the unfortunate situation that for most of the astrophysically interesting stars no accurate proper motions are available, while most of the stars with highest astrometric accuracy are of no astrophysical importance. 


\section{OUTLOOK ON REFERENCE FRAMES FOR GALACTIC ASTRONOMY}

In the near and medium future, I expect that conventional global reference systems will provide absolute proper motions of stars with increased systematic and individual accuracy. Two of these reference frames will be of special importance: (1) The Fifth Fundamental Catalogue, FK5, is being prepared at the Astronomisches Rechen-Institut Heidelberg from meridian circle observations covering more than eight decades. The basic FK5 shall be finished in 1986/1987. (2) The final results of observations with the planned European astrometric satellite HIPPARCOS should be available in 1993/1994. The HIPPARCOS system of proper motions itself will not be an inertial one, but it should be extremely rigid, thereby minimizing regional distortions. It is, however, expected that the HIPPARCOS system can be linked to quasars either via radio stars by using radio interferometers, or by measuring the motions of HIPPARCOS stars relative to quasars optically with the Hubble Space Telescope.

In the more distant future, I expect direct measurements of 'inertial proper motions' of some stars by space telescopes: In these measurements, the proper motion of a star will be measured relative to two neighbouring quasars. Such local measurements can be very accurate. In this way, we would completely avoid large-angle measurements as used in the FK5 and HIPPARCOS catalogues, for deriving inertial proper motions required for galactic astronomy. The main difficulty for galactic astronomy in this respect is the fact that most of the stars of interest are located in the galactic belt, which is unfortunately also the zone of avoidance for extragalactic objects including quasars.

\section{ON OBSERVATIONAL LIMITS FOR THE ROTATION OF THE EXTRAGALACTIC REFERENCE FRAME}

In the future, quasars and other extragalactic objects will provide the basic reference frame for proper motions. How accurately do quasars define an inertial system ? There do exist at least two independent ways of setting an upper limit on the rotation of the universe by observations: (1) Collins and Hawking (1973) have derived, from the absence of strong anisotropies in the $3 \mathrm{~K}$ background radiation, an upper limit for the rotation of the universe of $\omega_{\text {univ }}<10^{-10} \mathrm{n} /$ century $=10^{-9}$ marcsec/year. (2) Treder (1985) has shown that the constant of aberration depends on distance (i.e. on redshift) in a rotating universe. The absence of an obvious effect of this kind in VLBI measurements of neighbouring quasars with widely different values of redshift z (e.g. Marcaide and Shapiro 1983) would also indicate a very smal1

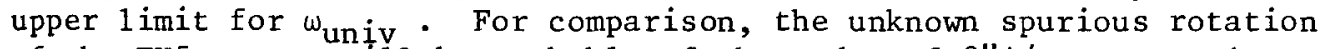
of the FK5 system will be probably of the order of $0.11 /$ century $=1$ marcsec/year. Hence, in comparison to conventional reference systems, quasars provide in principle a rather ideal inertial reference system for measuring proper motions of galactic objects. 


\section{REFERENCES}

Collins, C.B., Hawking, S.W.: 1973, Mon. Not. R. Astron. Soc. 162, 307. Marcaide, J.M., Shapiro, I.I.: 1983, Astron. J. 88, 1133.

Treder, H.-J.: 1985, Ann. Phys. (Leipzig), 7. Folge, Band 42, p.71. Wielen, R.: 1982, in 'The Scientific Aspects of the HIPPAR $\overline{C O S}$ Space Astrometry Mission', Eds. M.A.C. Perryman and T.D. Guyenne, Proc. Intern. Co11., Strasbourg, 22-23 February 1982, ESA SP-177, p. 77 . 